\title{
A Noniterative Approach for Computing Linear Equaliser Tap Weights
}

\author{
C Tellambura \\ Monash University, Dept. of Digital Systems \\ Clayton, Victoria 3168, Australia \\ Email: chintha@dgs.monash.edu.au \\ Y J Guo and S K Barton \\ Dept. of Electrical and Electronic Eng. \\ University of Bradford \\ West Yorkshire BD7 1DP, UK
}

\begin{abstract}
A fast computation method for the tap weights of a linear equaliser is developed. By approximating the channel autocorrelation matrix, which has a Toeplitz structure, by a circulant matrix, the tap weights can be solved via discrete fourier transform (DFT) techniques. For an equaliser with $N$ taps, the approximate tap weights approach the exact tap weights as $N \rightarrow \infty$. Excess mean-square error and bit error rate (BER) degradation caused by the approximate tap weights are computed for a representative channel.
\end{abstract}

\section{INTRODUCTION}

An equaliser is a filter that compensates intersymbol interference (ISI) in communication channels and is indispensable in some receivers such as high-speed modems. Consider a linear equaliser with the tap weight vector $\hat{\mathbf{c}}=\left[\hat{c}_{1}, \ldots, \hat{c}_{N}\right]^{\prime}$ where prime indicates transposition and $N$ is the number of taps (equaliser span). The cardinal question is, then, how to select $\hat{\mathbf{c}}$ in the face of unknown and possibly time-varying channel characteristics. Thus, the equaliser is 'trained' at the begining of each data transmission.

The mean-square error (MSE) criterion is commonly used for this purpose where the MSE is a convex function of $\hat{\mathbf{c}}_{n}$, giving rise to the existence of a unique global minimum. The optimum tap weights vector $\mathbf{c}_{\text {opt }}$ that minimizes the MSE is given by $[1,(6.4 .48)]$

$$
\mathbf{R c}_{\mathrm{opt}}=\mathbf{x}
$$

where $\mathbf{x}$ is the $N \times 1$ cross correlation vector between the equaliser input signal vector and the error signal and if the channel correlation matrix $\mathbf{R}=\left[r_{i, j}\right]$, then $r_{i, j}=\rho(i-j)$ where $\rho(x)$ is the channel correlation function (for details see [1]). In general, $\mathbf{R}$ is Hermitian and positive semidefinite (however, it does sometimes have the stronger property of positive definiteness). In addition, it is a Toeplitz matrix (for a symbol-spaced equaliser), i.e., equal elements along diagonals parallel to the principle diagonal. The optimum equaliser achieves the minimum MSE (MMSE) given by

$$
J_{\min }=1-\mathbf{c}_{\mathrm{opt}}^{H} \mathbf{R}^{-1} \mathbf{c}_{\mathrm{opt}}
$$

The work described in this paper was supported by the UK DTI/EPSRC LINK project: PC2011 "High Throughput Radio Modem" in collaboration with Symbionics Networks Limited under EPSRC grant GR/K00318. where the superscript $H$ indicates conjugate transposition.

To solve (1) in practice, almost invariably, a known training sequence is embeded in the signal, so that the difference between a locally synthesised copy of this sequence and the output of the equaliser can be used to derive an error signal to drive an iterative algorithm towards $c_{\text {opt }}$. For example, the least mean-square (LMS) algorithm is one of the most commonly used iterative algorithms in practice. However, owing to the use of noisy gradient estimates, the LMS algorithm only provides an approximation to $\mathbf{c}_{\mathrm{opt}}$. Nor can it attain the MMSE, $J_{\min }$. Moreover, its convergence rate is highly sensitive to the channel characteristics and decreases as $N$ increases. Despite these drawbacks, it is widely used for its simplicity and robustness.

Unlike the LMS algorithm, the noniterative (direct) method of equaliser training requires a full matrix inversion to solve (1). This approach can be divided into two distinct phases: estimating the channel impulse response (CIR) and solving for $\mathrm{c}_{\mathrm{opt}}$. While not denying the importance of channel estimation, this paper concentrates on solving for $\mathbf{c}_{\mathrm{opt}}$ given the CIR. CIR estimates can be obtained accurately using correlation techniques, least-squares filtering, and DFT methods with periodic estimation sequences [2].

At first glance the direct method appears computationally less efficient than the iterative approach, since matrix inversion (e.g., using Gaussian elimination) is an $O\left(N^{3}\right)$ process in general. In contrast, the LMS algorithm has complexity per iteration $O(N)$. However, since $\mathbf{R}$ is Toeplitz, using the Levinson-Trench algorithm, the direct method will only cost $O\left(N^{2}\right)$ [3]. Thus, much work has been done on developing direct equaliser training methods: for linear equalisers [3] and decision feedback equalisers (DFE) [4]. These rely on the Levinson-Trench algorithm, which involves exploiting the symmetric properties of $\mathbf{R}^{-1}$, arising out of $\mathbf{R}$ being Toeplitz, to reduce computation. Further, it involves an iterative solution of (1) for all its principal submatrices. To ensure stability, $\mathbf{R}$ and all its principle submatrices must be nonsingular (i.e., "strongly nonsingular"), in addition to being Toeplitz [5]. Implementing such algorithms can be tricky in practice. 
DFT based techniques for direct tap computation have also been derived before [6], [7]. These cyclic equalisation methods require a periodic training sequence whose period is equal to the equaliser span, $N$. This approach can be viewed as frequency domain equalisation in which, under noise-free conditions, the periodic equaliser inverts the channel frequency response at $N$ discrete frequencies. This will not attain the MMSE with random input data. In contrast, the DFT method developed here does not require any special training sequence and is not a frequency domain approach. It is based on the assumption that the channel span is short compared to the equaliser span. It is also possible to embed an $N \times N$ Toeplitz matrix in a $2 N \times 2 N$ circulant matrix, which can be used to solve a system of Toeplitz equations iteratively [8].

\section{FAST COMPUTATION OF TAP WEIGHTS}

Here it is assumed that the input signal autocorrelation function (ACF) has a finite duration, i.e., $\rho(k)=0$ for $|k| \geq L$. Clearly, $L$ is an indication of the channel memory. This assumption is quite reasonable in practice, and most channels are characterized by an exponentially-decaying impulse response. The relevant $\mathrm{ACF}$ is

$$
\rho(k)=\sum_{n} x(n) x^{*}(n-k)+N_{0} \delta(k)
$$

where $x(t)$ is the overall channel impulse response presented to the equaliser, the samples, taken at $T$ intervals where $T$ is the data symbol duration, are $x(n) \triangleq_{x}(n T)$, and $N_{0}$ is the additive noise spectral density. Here $x(t)$ is the convolution of the channel impulse response with transmitter and receiver pulse shaping. Any excess bandwidth used in raised-cosine filtering will also result in narrow pulses in the time domain, which can reduce the spread, $L$.

Under this assumption, $\mathbf{R}$ becomes a banded matrix, i.e., $r_{i j}=0$ for $|i-j| \geq L$. Now consider the case when $N \gg L$. It has been shown [9] that $\mathbf{R}$ can be approximated by a circulant matrix (the first row is sufficient to determine an entire circulant matrix, and each row is a cyclic shift of the preceding row). Let $C 1=\left[c_{0}, c_{1}, \ldots, c_{N-1}\right]$ denote the first row of the circulant matrix C. Choose $c_{k}=\rho(-k)$ for $k=0,1, \ldots, L-1$, $c_{k}=\rho(N-k)$ for $k=N-L+1, \ldots, N-1$, and $c_{k}=0$ otherwise. Then, $\mathbf{C}$ is asymptotically equivalent to $\mathbf{R}$ [9]. Therefore, instead of (1), the following should be solved:

$$
\mathrm{Cc}_{\mathrm{app}}=\mathrm{x}
$$

The idea of using the circulant approximation for tap computation was first proposed by Lee and Cioff [10] to develop a tap solution method for a frequency domain DFE. Moreover, they assumed that the noiseless channel output is periodic to ensure the correlation matrices being circulant. However, this paper is only concerned with linear MSE equalisers and the following development is quite different from theirs.

To establish the asymptotic equivalence between the

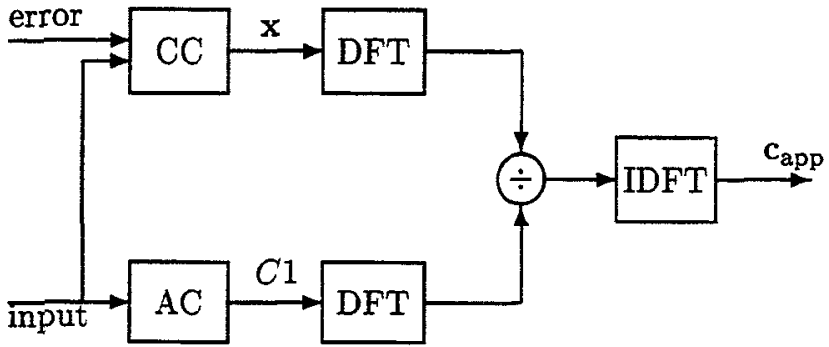

Fig. 1. DFT calculation of the tap weights. Cross Correlation (CC) and Auto Correlation (AC).

two solutions, i.e., for a given vector norm $\|$.$\| ,$

$$
\lim _{N \rightarrow \infty}\left\|\mathbf{c}_{\text {app }}-\mathbf{c}_{\text {opt }}\right\| \rightarrow 0
$$

provided $L / N \rightarrow 0$, consider $\mathbf{C}$ as a perturbation of $\mathbf{R}$. The inherent relative error is then bounded as [11]

$$
\frac{\left\|\mathbf{c}_{\text {app }}-\mathbf{c}_{\text {opt }}\right\|}{\left\|\mathbf{c}_{\text {opt }}\right\|} \leq \frac{\left\|\mathbf{R}^{-1}(\mathbf{C}-\mathbf{R})\right\|}{1-\left\|\mathbf{R}^{-1}(\mathbf{C}-\mathbf{R})\right\|}
$$

provided $\left\|\mathbf{R}^{-1}(\mathbf{C}-\mathbf{R})\right\|<1$. Since $\mathbf{C}$ is identical to $\mathbf{R}$ everywhere except the upper right hand and lower left hand corners, there exists [9] a $K<\infty$ such that $\|(\mathbf{C}-\mathbf{R})\| \leq K N^{-1 / 2}$. Further assume that $\left\|\mathbf{R}^{-1}\right\|<$ $\infty$ (well-conditioned). Combining these two inequalities yields the limit above.

Having established the asymptotic equivalence, consider how $c_{a p p}$ can be computed. A circulant matrix has the decomposition

$$
\mathrm{C}=P_{N}^{*} \Lambda P_{N}
$$

where $P_{N}$ is the $N \times N$ Fourier matrix ${ }^{1}$ with $p_{r, s}=\exp (-j 2 \pi r s / N) / \sqrt{N}, j=\sqrt{-1}$, and $r, s=0,1, \ldots, N-1$. And the eigenvalue matrix $\Lambda=\operatorname{diag}\left(\lambda_{0}, \lambda_{1}, \ldots, \lambda_{N-1}\right)$ where $\left[\lambda_{0}, \lambda_{1}, \ldots, \lambda_{N-1}\right]^{\prime}=$ $P_{N} C 1^{\prime}$, the DFT of the first row. Thus, the tap weights are given as

$$
\mathrm{c}_{\mathrm{app}}=P_{N}^{*} \Lambda^{-1} P_{N} \mathbf{x}
$$

Here the existence of $\Lambda^{-1}$ (see below) is assumed. Thus the sequence of operations necessary for computing $\mathbf{c}_{\text {app }}$ is as follows: compute DFTs of $C 1$ and $\mathrm{x}$, perform element-wise vector division, and take the IDFT. Fig. 1 shows all the steps involved. Since in general computing the DFT or IDFT is an $O(N \log N)$ process, this method will also cost $O(N \log N)$.

\section{A. Asymptotic eigenvalues}

As an aside, the eigenvalues of $\mathbf{R}$ and $\mathbf{C}$ coincide as $N \rightarrow \infty$ [9]. Based on this fact, it can be shown that

\footnotetext{
${ }^{1}$ That means, for an $N \times 1$ vector $\mathbf{z}$, its DFT and inverse DFT (IDFT) are $P_{N} \mathrm{z}$ and $P_{N}^{*} \mathrm{z}$, respectively
} 
[12] the eigenvalues of $\mathbf{C}$ are given by the samples of the aliased or folded spectrum

$$
R(\omega)=\frac{1}{T}\left|\sum_{n} X\left(\omega+\frac{2 \pi n}{T}\right)\right|^{2}+N_{0} \quad|\omega| \leq \frac{\pi}{T}
$$

where $X(\omega)$ is the Fourier transform of $x(t)$, defined in (3). Let $N=2 K+1$ and the equi-spaced frequencies $\omega_{i}=2 \pi i / N T,-K \leq i \leq K$, sample the Nyquist bandwidth. Thus, as $N \rightarrow \infty$ the $N$ eigenvalues of $\mathbf{R}$ are given by

$$
\lambda_{i}=R\left(\omega_{i}\right) \quad i=-K, \ldots, K .
$$

In other words, the $N$ equi-spaced samples of the folded spectrum in the Nyquist band $(|\omega|<\pi / T)$ are the eigenvalues (this analysis matches with that given in [13]). Thus, any null in the folded spectrum, caused by nulls in the overall channel response, $X(\omega)$, or cancellation due to spectral aliasing, will result in some eigenvalues that are nearly zero (as $N_{0} \rightarrow 0$ ). Since the convergence rate of the LMS and other iterative algorithms is determined by the minimum eigenvalue, which is now nearly zero, these algorithms will exhibit poor convergence rates in such a channel [1]. Conversely, when noise is present the eigenvalues are always positive (i.e., positive definite R). Since $x(t)$ is the overall impulse response presented to the equaliser, any sampling time errors can be incorporated into it. Thus, let the timing error be $t_{0}$, i.e., samples are taken at $n T+t_{0} \forall n$. Then, the folded spectrum becomes

$$
R(\omega)=\frac{1}{T}\left|\sum_{n} X\left(\omega+\frac{2 \pi n}{T}\right) \exp j\left(\omega+\frac{2 \pi n}{T}\right) t_{0}\right|^{2}+N_{0}
$$

for $|\omega| \leq \pi / T$. The summation should be done before taking the magnitude. This means depending on the sampling phase, $t_{0}$, spectral nulls can occur in the folded spectrum, especially near the band edges $(|\omega| \approx \pi / T)$ in excess-bandwidth systems. This cancellation is more likely to occur as $t_{0} \rightarrow T / 2$, which can result in small or nearly-zero eigenvalues. Thus, for the synchronous equaliser, the eigenvalues can be sensitive to the timing phase, which explains its poor performance observed in the presence of such errors [14]. In fact, assuming the excess bandwidth is less than $100 \%$, let the fractional excess bandwidth be $0<\alpha<1$, i.e., $|X(\omega)|=0$ for $|\omega| \geq(1+\alpha) \pi / T$. Thus,

$$
R(\omega)=\left\{\begin{array}{c}
\frac{1}{T}|X(\omega)|^{2}+N_{0} \\
\frac{1}{T}\left|X(\omega)+X\left(\omega \pm \frac{2 \pi}{T}\right) \exp j\left(\omega \pm \frac{2 \pi}{T}\right) t_{0}\right|^{2}+N_{0}
\end{array}\right.
$$

where the first expression is for $|\omega| \leq(1-\alpha) \pi / T$ and the second for $|\omega| \geq(1-\alpha) \pi / T$. The minus signs in the lower expression corresponds to the positive rolloff region, $\pi / T>\omega>(1-\alpha) \pi / T$, and the plus signs to the other side. Thus, we see that $(1-\alpha) N$ eigenvalues are not sensitive to the timing errors, but $\alpha N$ eigenvalues are. Thus, the larger the excess bandwidth, the worse the effect of timing errors.

Finally, the DFT based solution (7) is not immune to the problems caused by small eigenvalues. A possible solution is to add a small positive quantity to the diagonal terms of $\mathbf{R}$, which is akin to replacing $N_{0}$ by $N_{0}+\Delta$. This is obviously called "prewhitening" and results in a well-conditioned system of equations, but introduces some performance penalty. Usually, the correction quantity is less than $5 \%$ of $\rho(0)$ [15].

Any mismatch between $\mathbf{c}_{\text {app }}$ and $\mathbf{c}_{\text {opt }}$ is likely to manifest as excess MSE and BER degradation. These are addressed in the next two subsections.

\section{B. Excess MSE}

Since $c_{a p p} \neq c_{\mathrm{opt}}$, the approximate equaliser cannot attain the MMSE in (2). The excess MSE can readily be shown to be

$$
J_{\mathrm{ex}}=\mathrm{c}_{\mathrm{app}}^{H} \mathbf{R} \mathbf{c}_{\mathrm{app}}-\mathbf{c}_{\mathrm{app}}^{H} \mathbf{x}-\mathbf{x}^{H} \mathbf{c}_{\mathrm{app}}+\mathbf{x}^{H} \mathbf{R}^{-1} \mathbf{x} .
$$

If $c_{\text {app }}=c_{\text {opt }}$, then $J_{\mathrm{ex}}=0$.

Fig. (2) shows $10 \log _{10}\left(J_{\text {ex }}\right)$ for a linear equaliser in a typical data-quality telephone channel. The CIR used is that given by the discrete channel tap weights $\left\{f_{k}\right\}$ found in [1, Fig. 10-2-5(a)] and the channel span is 11 symbols. The equaliser input signal-to-noise ratio (SNR) is defined as $\gamma=\sum\left|f_{k}\right|^{2} / N_{0}$ where $N_{0}$ is the additive noise level. Even for $N=2 L$, the excess MSE is less than $-20 \mathrm{~dB}$. At $N \approx 3 L$, the excess MSE is $-40 \mathrm{~dB}$. These small values show that the performance with $c_{\text {app }}$ is indeed very close to that with $\mathbf{c}_{\mathrm{opt}}$. Also, the excess MSE decays very rapidly for low $\gamma$ values, indicating the improved accuracy at low SNRs. This is because, for low $\gamma, N_{0}$ is relatively large, which imparts impulselike characteristics to $\rho(x)$. In other words, for large $N_{0}$, noise swamps the signal. Then, as the ACF of noise is an impulse function, $\mathbf{R}$ approaches a diagonal matrix (i.e., already approximately circulant).

\section{BER degradation}

Since the BER of an equalised system is a highly nonlinear function of the tap weights, the effect of using $\mathbf{c}_{\text {app }}$ instead of $\mathbf{c}_{\text {opt }}$ must be estimated via simulation or numerical computation. Also, the MMSE system is not guaranteed to give the minimum BER receiver in general. However, for binary systems, an upper bound on the BER monotonically decreases with the MSE [16]. Thus, any excess MSE may be expected to translate into a BER degradation.

For a data sequence $I_{n}$, the equaliser output can be written as [1, eq. (10-2-56)]

$$
\hat{I}_{n}=q_{0} I_{n}+\sum_{k \neq n} I_{k} q_{n-k}+\eta_{n}
$$

where $\left\{q_{n}\right\}$ denote the convolution of the impulse response of the equaliser and equivalent channel, and $\eta_{n}$ the filtered additive noise. Thus, for a given channel $\left\{q_{n}\right\}$ can be computed for $\mathrm{c}_{\mathrm{opt}}$ or $\mathrm{c}_{\mathrm{app}}$. Since the output contains a non-Gaussian term, the BER cannot be computed in terms of the Gaussian tail probabilities. An infinite series developed by Bealieu [17, eq. (30)] has been used for this purpose. Fig. 3 shows the BER performance for the approximate and exact equalisers with BPSK modulation over the same channel used in the 
previous example. Again at $N \approx 3 L$, there is virtually no difference between the performance of the two equalisers.

\section{Channel estimation}

Thus far it was assumed that the CIR is known at the receiver. In practice, however, it must be estimated. Since the approximate solution (7) leads to degraded performance (albeit a negligible loss with a perfect CIR estimate), channel estimation errors are likely to exacerbate such a performance loss. To delineate the effect of channel estimation errors and of the circulant approximation, an example is provided here. The same 11 tap channel is used again with an $m$-sequence based channel estimator. As shown in [18], $m$-sequences are not the most efficient for this purpose ${ }^{2}$. However, they are characterized by a two-valued ACF, which leads to a very simple realization of the channel estimator. In fact, computational complexity is just $O(L)$ to estimate $L$ channel tap weights (independent of the $m$-sequence length), and in some cases, the channel estimator is completely multiplication-free, requiring only additions. Fig. 4 shows the results for two $m$-sequences of lengths 31 and 63 bits under varying input SNR, $\gamma$, which is identical for both the channel estimator and 31 tap equaliser. For comparison, the BER when perfect knowledge of the CIR is available is also given. The BER with channel estimation is ensemble-averaged over the noise samples presented to the channel estimator. The noise power, $N_{0}$, itself is estimated at the input to the equaliser. For $m=63$, and for BER of $10^{-5}$, the use of channel estimates leads to a loss of about $0.5 \mathrm{~dB}$ and the use of the approximation (7) leads to a further loss of about 0.5 $\mathrm{dB}$. These losses decrease for high BERs (equivalently, for low SNRs), but increase for the case of $m=31$ channel estimator. Thus, it might be concluded that $m=31$ is inadequate in this case. Also, although it has 11 taps, this channel is a quite benign ISI channel and hence, the equaliser can operate at low SNRs (e.g., 12dB). In contrast, the channel estimator works best at high SNRs, in which case $m=31$ should be sufficient.

\section{CONCLUSIONS}

This paper has shown that a circulant approximation provides an efficient direct computation of the tap weights of a linear equaliser. Although the spread of the input signal ACF must be small compared to the equaliser span for this method to work best, the tap weights can be improved by decision-directed equalisation to achieve the optimum tap settings. The precomputation of eigenvalues highlights those values which are small or nearly-zero, giving prior warning of overlarge tap weights and dynamic range. From an implementation point of view, DFT based solutions are attractive because they can be implemented with off-the-shelf hardware. Finally, the approximate method can be extended to the case of fractionally-spaced equalisers [12].

\footnotetext{
${ }^{2}$ Here most efficient implies yielding the smallest mean-squared estimation error for a given sequence length $N$, say, for estimating $L$ channel taps. Thus, shorter binary sequences can be found to yield the same efficiency as a longer $m$-sequence, but the efficiency disparity diminishes as $L / N \rightarrow 0$.
}

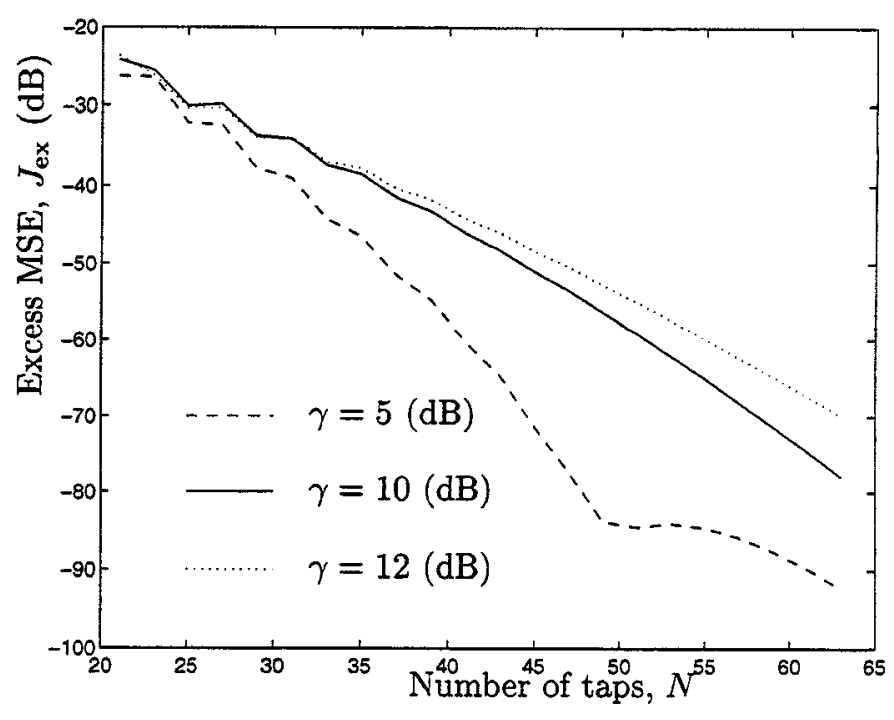

Fig. 2. The excess MSE due to the use of $\mathbf{c}_{\text {app. }}$.

\section{REFERENCES}

[1] J. G. Proakis, Digital Communications. New York: McGraw Hill, 3rd ed., 1995.

[2] B. Steiner and P. Jung, "Optimum and suboptimum channel estimation for the uplink of CDMA mobile radio systems with joint detection," Euro. Trans. Telecom., vol. 5, pp. 39-50, Jan.-Feb. 1994.

[3] P. Butler and A. Cantoni, "Noniterative automatic equalization," IEEE Trans. Commun., vol. COM-23, pp. 621-633, June 1975.

[4] P. Shukla and L. Turner, "Channel-estimation based adaptive DFE for fading multipath radio channels," IEE Proc. $I$ vol. 138, pp. 525-543, Dec. 1991.

[5] S. Zohar, "The solution of a Toeplitz set of linear equations," J. Ass. Comput. Mach., vol. 21, pp. 272-276, 1974.

[6] K. H. Mueller and D. A. Spaulding, "Cyclic equalizationa new rapidly convverging equalization technique for synchronous data communication," Bell Syst. Tech. J., vol. 54, pp. 369-406, Feb. 1975.

[7] S. U. H. Qureshi, "Fast start-up equalization with periodic training sequences," IEEE Trans. Inform. Theory., vol. IT23, pp. 553-563, Sept. 1977.

[8] R. Yarlagadda and B. N. Suresh Babu, "A note on the application of FFT to the solution of a system of Toeplitz normal equations," IEEE Trans. Cir. and Sys., vol. CAS-27, pp. 151154, Feb. 1980.

[9] R. M. Gray, "On the asymptotic eigenvalue distribution of Toeplitz matrices," IEEE Trans. Inform. Theory., vol. IT18, pp. 725-730, Nov. 1972.

[10] I. Lee and J. M. Cioff, "A fast computation algorithm for the decision feedback equalizer," IEEE Trans. Commun., vol. 43, pp. 2742-2749, Nov. 1995.

[11] G. W. Stuart, Introduction to matrix computations. Academic Press, INC., 1973.

[12] C. Tellambura, Y. J. Guo and S. K. Barton, "Fast noniterative computation of linear equalizer tap weights," 1996. submitted to IEEE Trans. Commun.

[13] R. D. Gitlin and S. B. Weinstein, "On the required tap-weight precision for digitally implemented, adaptive, mean-squared equalizersr," Bell Syst. Tech. J., vol. 58, pp. 301-321, Feb. 1979

[14] S. U. Qureshi, "Adaptive equalization," IEEE Proc., vol. 73, pp. 1349-1387, Sept. 1985.

[15] P. M. Clarkson, Optimal and adaptive signal processing. CRC Press, Inc., 1993.

[16] B. R. Saltzberg, "Intersymbol interference error bounds with applications to ideal bandlimited signalling," IEEE Trans. Inform. Theory., vol. IT-14, pp. 563-568, July 1968.

[17] N. C. Bealieu, "The evaluation of error probabilities for intersymbol and cochannel interference," IEEE Trans. Commun. vol. 39, pp. 1740-1749, Dec. 1991.

[18] S. N. Crozier, D. D. Falconer, and S. A. Mahmoud, "Least sum of squared errors (LSSE) channel estimation," IEE Proceedings- $F$, vol. 138, pp. 371-378, Aug. 1991. 


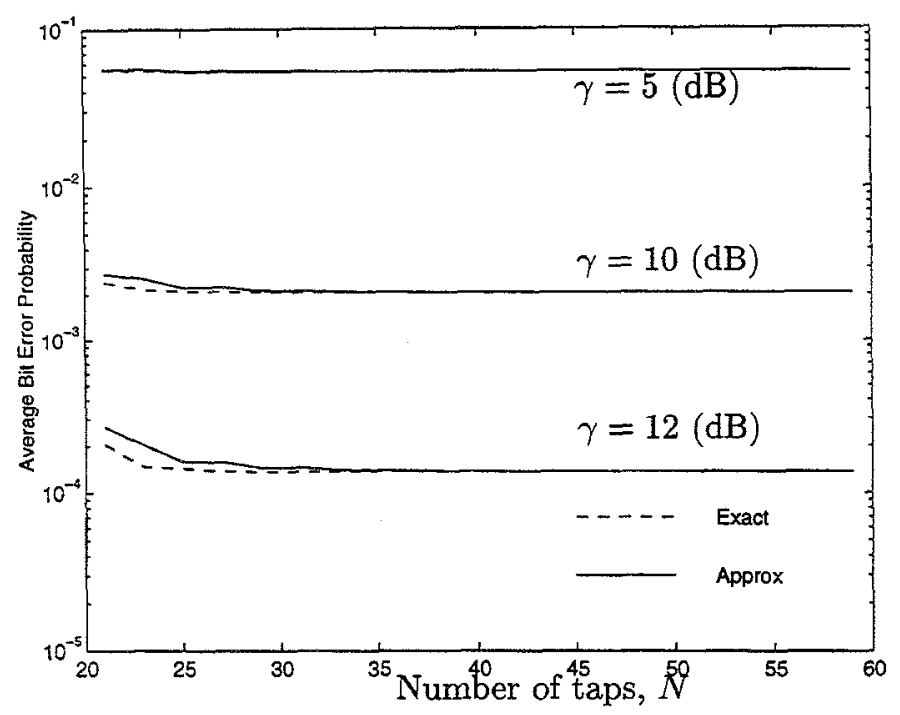

Fig. 3. The average bit error probability of an $N$ tap equaliser with $c_{\text {opt }}$ (Exact) or $c_{a p p}$ (Approx) tap weights. BPSK modulation is used.

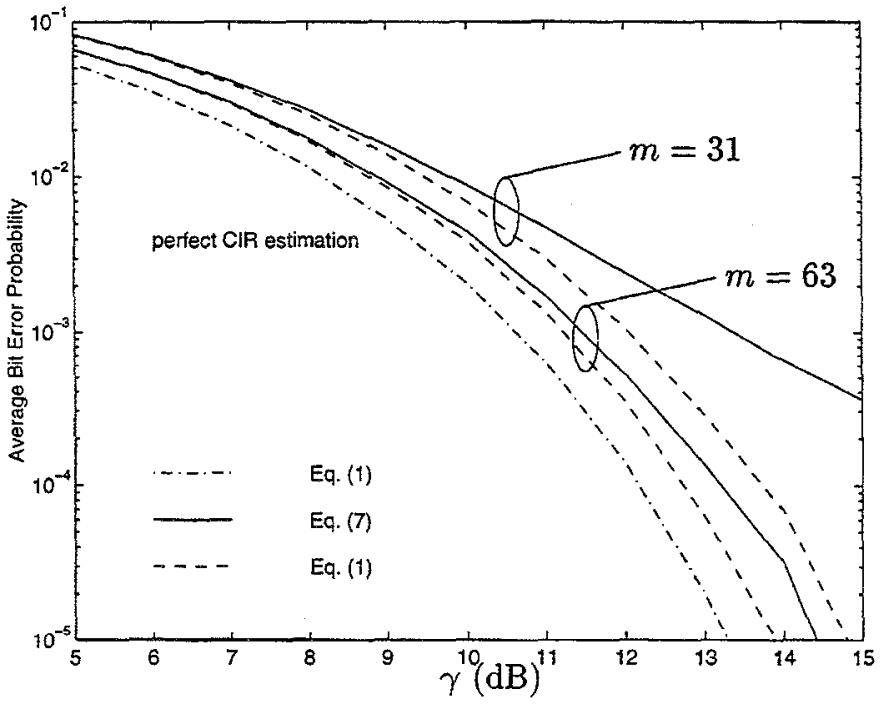

Fig. 4. The average bit error probability of a 31 tap equaliser with an $m$-sequence channel estimator. BPSK modulation is used. 